NFPI-10-1

\title{
The Michelson-Morley experiment in an accelerated reference frame
}

\author{
Dennis Crossley* \\ Dept. of Physics, University of Wisconsin-Sheboygan, Sheboygan, WI 53081
}

\begin{abstract}
We analyze the Michelson-Morley experiment in a reference frame moving with constant proper acceleration. Interestingly, we find an expected fringe shift which depends not only on the interferometer's rate of acceleration, but also on its speed relative to a preferred absolute reference frame. While it has been repeatedly shown that no experiment performed in an inertial reference frame can detect that frame's absolute speed, the analysis in this paper suggests that by considering experiments in accelerated reference frames it may be possible to measure absolute speed after all.
\end{abstract}

Keywords: special relativity, Michelson-Morley experiment, accelerated reference frame, absolute space

PACS: 03.30.+p

\section{Introduction}

The Michelson-Morley experiment [1] is one of the classic experiments in the history of physics. Its original purpose was to measure the speed of the earth through the luminiferous ether by measuring the shift of interference fringes in an interferometer. The ether was, at the time, believed to be the medium through which light waves traveled at speed $c$. It was assumed that the earth was moving at some speed $v$ relative to the lumiferous ether. The only property of the ether that concerns us here is that it is a unique preferred reference frame in which light travels at the same speed in all directions. In our analysis we will make reference to this absolute reference frame and leave all references to the luminiferous ether to the past.

Fig. 1 1 shows the light paths through the interferometer. The experiment involves sending a beam of light from a source $s$, splitting the beam at $a$ so that it follows two perpendicular paths $a b d$ and $a c d$, recombining the beams at $d$, and observing the resulting interference fringes at $o$. A classical calculation shows that the motion

\footnotetext{
*dennis.crossley@uwc.edu
} 


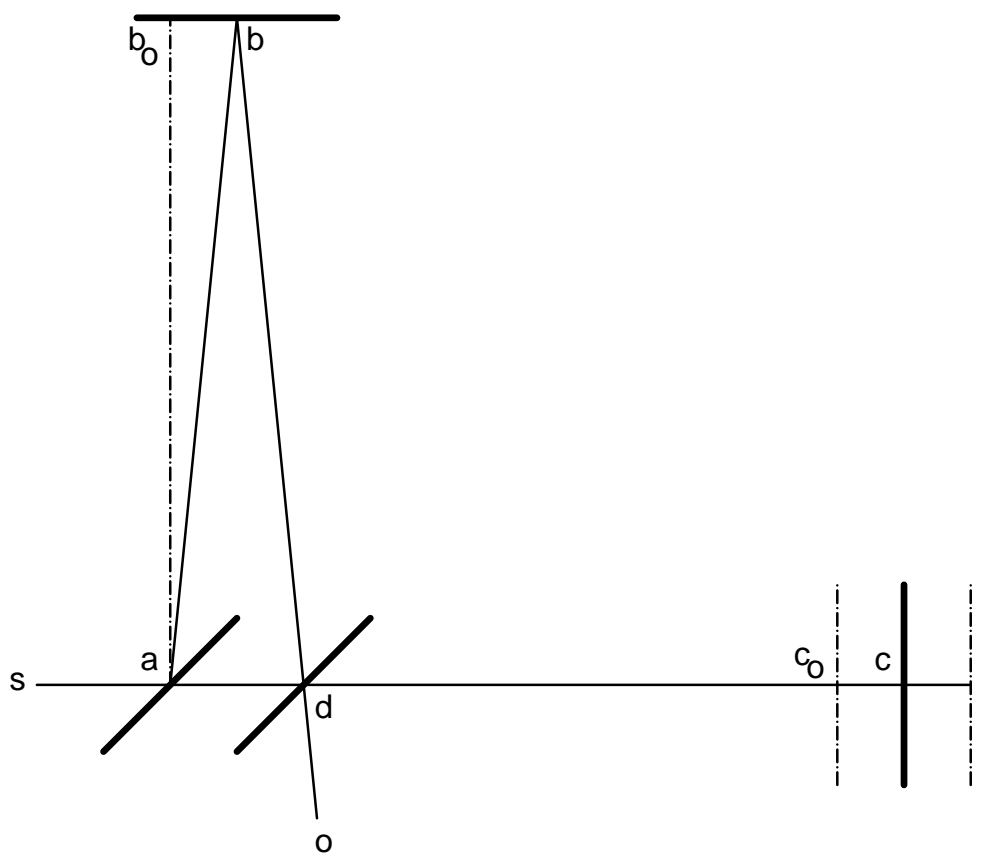

Figure 1: Light paths in the Michelson-Morley interferometer.

of the earth at speed $v$ relative to the absolute reference frame should cause the light traveling along a path parallel to the direction of motion to take slightly longer than light traveling along a path perpendicular to the direction of motion, the difference in travel time being

$$
\Delta t=\frac{L}{c} \cdot \frac{v^{2}}{c^{2}}
$$

to second order in $v / c$; $\mathrm{L}$ is the length of the interferometer arms. By rotating the interferometer through ninety degrees, Michelson expected to see a net shift of interference fringes equal to 0.4 fringe. What he measured was at most 0.01 fringe shift. This is generally interpreted as a null result.

The analysis of this experiment is based on two implicit assumptions: (1) there exists an absolute reference frame in which light travels at a constant speed $c=$ $3.00 \times 10^{8} \mathrm{~m} / \mathrm{s}$ relative to this reference frame, and (2) the geometry of the interferometer is not changed by its motion. These assumptions play a pivotal role in the two alternative explanations for the null result obtained in the experiment.

The first explanation was proposed by Fitzgerald[2] and Lorentz[3]. They chose to abandon the second assumption. They pointed out that if the arm of the interferometer in the direction parallel to the direction of motion shrank by a factor of 
$\sqrt{1-v^{2} / c^{2}}$ that the expected fringe shift would be exactly canceled, resulting in the observed null result. They interpreted this shortening of the interferometer arm as an actual physical shortening. Although Lorentz gave a reasonable justification for this physical shortening in terms of his theory of the electron, this explanation was generally considered $a d h o c$ and did not gain general acceptance.

The second explanation was presented by Einstein who chose on philosophical grounds to abandon the first assumption, simply assuming the non-existence of an absolute reference frame. It is an important point, however, that the special theory of relativity, though consistent with the non-existence of an absolute reference frame, does not prove this non-existence, only the impossibility of detecting it from an inertial reference frame 4 .

The traditional analysis of the Michelson-Morley experiment assumes that the observer and his interferometer are in an inertial reference frame, moving at a constant speed $v$ relative to the absolute reference frame. The failure of the traditional analysis of the Michelson-Morley experiment to measure this speed, and the subsequent success of special relativity, which raises the constancy of the speed of light to the level of a fundamental postulate, demonstrate the difficulty of measuring the observer's absolute speed by performing any experiment in an inertial reference frame.

But it is well known that while constant velocity motion is relative, accelerated motion is absolute. Two space travelers can be accelerating relative to each other while only one (the one with his rockets firing) is pushed back into his seat. The ability to detect absolute acceleration mechanically suggests the possibility of detecting absolute acceleration optically as well using, for example, a MichelsonMorley interferometer. This idea gains support from statements made by Desloge and Philpott [5],

In an inertial frame, distances measured with standard rods and distances made with light signals give identical results. Furthermore, the clocks of the individual observers making up an inertial frame can be synchronized and will remain synchronized. Neither of the above facts is true for an accelerated frame.

and by Giannoni and Grøn[6],

We see that these two situations, one with acceleration of the clocks and the other with the acceleration of the observer, which are identical as to the relative movements between clocks and observer, nevertheless are physically different. In the former case, the clocks do not maintain their Einstein synchronization, while in the latter case they do so. This 
illustrates that absolute acceleration has an empirical and purely kinematical significance in the special theory of relativity which is lacking in Newtonian physics.

More simply, since it is well established that material objects cannot travel faster than the speed of light, a boost given to a moving object in the forward direction will produce a smaller change of velocity than a boost in the backward direction. In the Michelson-Morley experiment light travels in both the forward and backward directions and the degree to which the acceleration of the interferometer's reference frame affects the forward and backward travel times depends on the speed of the interferometer. The resulting fringe shift in the interferometer therefore depends not only on the interferometer's acceleration but also on its absolute velocity. This makes it possible, in principle at least, to measure absolute velocity using an interferometer in an accelerated reference frame.

\section{Kinematics of a Michelson-Morley interferometer in an accelerated reference frame}

In this paper we make the assumptions that (1) a preferred reference frame exists in which the speed of light is uniform in all directions, and (2) length contraction is a physically real phenomenon and depends on an object's speed relative to this preferred reference frame. The first assumption has found support in recent work by Cahill and Kitto[7]; and the second in the book by Brown[8] and a recent paper by Barcelò and Jannes [9]. We analyze a Michelson-Morley type experiment performed in a reference frame which is accelerating relative to this preferred reference frame at a rate which is constant relative to instantaneously co-moving inertial frames, otherwise known as constant proper acceleration.

Reference frames with constant acceleration relative to instantaneously co-moving inertial frames have been thoroughly investigated [10, 11, 12, 13, 15]. The motion of a point object with constant proper acceleration $g$ is referred to as hyperbolic motion and is described by the equation

$$
x^{2}-c^{2} t^{2}=\frac{c^{4}}{g^{2}}
$$

where the initial conditions $x=c^{2} / g$ and $v=0$ at time $t=0$ have been chosen. Thus the position of this point object as a function of time is

$$
x(t)=\sqrt{\frac{c^{4}}{g^{2}}+c^{2} t^{2}} .
$$


The velocity of this point object as a function of time is

$$
v(t)=\frac{g t}{\sqrt{1+\left(\frac{g t}{c}\right)^{2}}},
$$

which is easily inverted to get $t$ as a function of $v$,

$$
t(v)=\frac{v}{g \sqrt{1-\left(\frac{v}{c}\right)^{2}}} .
$$

This last equation will be useful for explicitly showing the velocity dependence of our results.

For an extended object, such as the arm of a Michelson-Morley interferometer, the different points of the object along the direction of motion will necessarily have different accelerations because of the effects of relativistic length contraction as the object's velocity changes. The various points along an extended object move along a family of hyperbolas described by the equation

$$
x^{2}-c^{2} t^{2}=X^{2},
$$

where $\mathrm{X}$ is a parameter identifying the location of each point at $t=0$.

When we consider the Michelson-Morley experiment in an accelerated reference frame, we must therefore be careful to identify which point of the interferometer is moving with constant proper acceleration. We choose the beamsplitter as this point. If we orient the interferometer so that it is moving in the positive $x$ direction (to the right in Fig. 1), with one arm parallel to the direction of motion and ahead of the beamsplitter, then the left end of the parallel arm and both ends of the perpendicular arm share the same $x$ coordinate and have motion described by the equation

$$
x_{L}^{2}-c^{2} t^{2}=\frac{c^{4}}{g^{2}}
$$

or

$$
x_{L}(t)=\sqrt{\frac{c^{4}}{g^{2}}+c^{2} t^{2}} .
$$

The right end of the parallel arm with proper length $L$ will be located at $x=c^{2} / g+L$ at $t=0$. Its motion will be described by the equation

$$
x_{R}^{2}-c^{2} t^{2}=\left(\frac{c^{2}}{g}+L\right)^{2},
$$

or

$$
x_{R}(t)=\sqrt{\left(\frac{c^{2}}{g}+L\right)^{2}+c^{2} t^{2}} .
$$


We assume that both arms have proper length $L$ and we can therefore identify the coordinates of the three key points of the interferometer as functions of time (as measured in the rest frame): beamsplitter at $\left(x_{L}(t), 0\right)$, far end of perpendicular arm at $\left(x_{L}(t), L\right)$, and far end of the parallel arm at $\left(x_{R}(t), 0\right)$.

Light entering a Michelson-Morley interferometer will, in general, return to the beamsplitter at different times depending on the travel times along the two arms. The difference between the return times can be used to calculate the phase shift in an interferometer moving with constant velocity but this method introduces a small error in the accelerated case. When an experiment is performed, interference is observed between the two lightbeams returning to the beamsplitter at the same time. We take this time, which we will call $t_{m}$ (time of measurement), as our reference time and calculate backwards to find the difference in times of entry into the beamsplitter. Besides giving a rigorously correct calculation of the travel time difference, this also allows us to identify unique values of the standard relativistic parameters $\beta$ and $\gamma$ as those pertaining to the time $t_{m}$,

$$
\beta=\frac{v\left(t_{m}\right)}{c}, \quad \gamma=\left(1-\beta^{2}\right)^{-\frac{1}{2}} .
$$

There are no restrictions on the value of $t_{m}$ and we want to consider the general case with $t_{m} \neq 0$ such that the interferometer has a nonzero velocity given by equation (4). Furthermore, we want to consider $t_{m}$ as a parameter and to solve for light travel times in terms of $t_{m}$, thereby encoding the velocity dependence of the result. After solving for the travel times along each arm, we will make this velocity dependence explicit by using equation (5).

\section{Michelson-Morley experiment in an accelerated ref- erence frame}

With the coordinates of the endpoints of the arms of a Michelson-Morley interferometer (moving with constant proper acceleration) identified as functions of time, we can now analyze the Michelson-Morley experiment in this accelerated reference frame.

We consider first light traveling along the perpendicular arm, with light entering the beamsplitter at point $a$ at time $t_{1}$, being reflected from the far mirror at point $b$ at time $t_{3}$, and returning to the beamsplitter at point $d$ at time $t_{m}$. Working backward in time, light traveling from $b$ to $d$ takes time $c\left(t_{m}-t_{3}\right)$. Time $t_{3}$ therefore must satisfy the equation

$$
c^{2}\left(t_{m}-t_{3}\right)^{2}=L^{2}+\left(x_{L}\left(t_{m}\right)-x_{L}\left(t_{3}\right)\right)^{2}
$$


This can be written as an equation in $t_{3}$ using equation (8),

$$
c^{2}\left(t_{m}-t_{3}\right)^{2}=L^{2}+\frac{c^{4}}{g^{2}}\left[\sqrt{1+\left(\frac{g t_{m}}{c}\right)^{2}}-\sqrt{1+\left(\frac{g t_{3}}{c}\right)^{2}}\right]^{2} .
$$

The solution of this equation for time $t_{3}$ is quite intricate, so we give an outline of the solution here. We introduce the (small) dimensionless parameters

$$
T_{i}=\frac{g t_{i}}{c} \quad \text { and } \quad \alpha=\frac{g L}{c^{2}},
$$

which transforms equation (13) into a dimensionless equation,

$$
\left(T_{m}-T_{3}\right)^{2}=\alpha^{2}+\left[\sqrt{1+T_{m}^{2}}-\sqrt{1+T_{3}^{2}}\right]^{2} .
$$

It is convenient to also define the quantities

$$
X_{i}=1+T_{i}^{2} \quad(i=1,2,3, m),
$$

which simplifies the equation even further,

$$
\left(T_{m}-T_{3}\right)^{2}=\alpha^{2}+\left(X_{m}^{1 / 2}-X_{3}^{1 / 2}\right)^{2} .
$$

By expanding the last term, rearranging, and squaring the resulting equation, we get a polynomial equation in $T_{3}$ of degree 4 ,

$$
\left[X_{m}+X_{3}-\left(T_{m}-T_{3}\right)^{2}+\alpha^{2}\right]^{2}-4 X_{m} X_{3}=0 .
$$

This quartic equation is then solved using a power series expansion of $T_{3}$ in powers of $\alpha$, solving for the coefficients of this power series (which are functions of $T_{m}$ ), then grouping terms into recognizable power series involving $\alpha$ and $T_{m}$. The result of this is the exact solution of equation (18),

$$
T_{3}=T_{m}-\alpha \sqrt{1+T_{m}^{2}} \sqrt{1+\left(\frac{\alpha}{2}\right)^{2}}+\frac{1}{2} T_{m} \alpha^{2} .
$$

To restore dimensioned quantities we observe that, using equation (5),$\sqrt{1+T_{m}^{2}}=\gamma$ and $T_{m}=\beta \gamma$, and we get

$$
t_{3}=t_{m}-\gamma \frac{L}{c} \sqrt{1+\left(\frac{\alpha}{2}\right)^{2}}+\beta \gamma \frac{L}{c}\left(\frac{1}{2} \alpha\right) .
$$

Light traveling from point $a$ to point $b$ takes time $c\left(t_{3}-t_{1}\right)$. Time $t_{1}$ therefore must satisfy the equation

$$
c^{2}\left(t_{3}-t_{1}\right)^{2}=L^{2}+\left(x_{L}\left(t_{3}\right)-x_{L}\left(t_{1}\right)\right)^{2},
$$


which can be written as an equation in $t_{1}$ using equation (8),

$$
c^{2}\left(t_{3}-t_{1}\right)^{2}=L^{2}+\frac{c^{4}}{g^{2}}\left[\sqrt{1+\left(\frac{g t_{3}}{c}\right)^{2}}-\sqrt{1+\left(\frac{g t_{1}}{c}\right)^{2}}\right]^{2} .
$$

Following a procedure similar to the one above, we convert this to a dimensionless equation,

$$
\left(T_{3}-T_{1}\right)^{2}=\alpha^{2}+\left(X_{3}^{1 / 2}-X_{1}^{1 / 2}\right)^{2}
$$

expand the last term, rearrange, and square the resulting equation to get a polynomial equation in $T_{1}$ of degree 4 ,

$$
\left[X_{3}+X_{1}-\left(T_{3}-T_{1}\right)^{2}+\alpha^{2}\right]^{2}-4 X_{3} X_{1}=0 .
$$

The exact solution of equation (24) is

$$
T_{1}=T_{3}-\alpha \sqrt{1+T_{m}^{2}}\left(1+\alpha^{2}\right) \sqrt{1+\left(\frac{\alpha}{2}\right)^{2}}+\frac{3}{2} T_{m} \alpha^{2}+\frac{1}{2} T_{m} \alpha^{4},
$$

which in dimensioned quantities gives

$$
t_{1}=t_{3}-\gamma \frac{L}{c}\left(1+\alpha^{2}\right) \sqrt{1+\left(\frac{\alpha}{2}\right)^{2}}+\beta \gamma \frac{L}{c}\left(\frac{3}{2} \alpha+\frac{1}{2} \alpha^{3}\right) .
$$

The total light travel time along the perpendicular arm is $t_{p e r p}=t_{m}-t_{1}$ :

$$
t_{\text {perp }}=\gamma \frac{L}{c}\left(2+\alpha^{2}\right) \sqrt{1+\left(\frac{\alpha}{2}\right)^{2}}-\beta \gamma \frac{L}{c}\left(2 \alpha+\frac{1}{2} \alpha^{3}\right) .
$$

Now consider light traveling along the parallel arm, with light entering the beamsplitter at point $a$ at time $t_{2}$, being reflected from the far mirror at point $c$ at time $t_{4}$, and returning to the beamsplitter at point $d$ at time $t_{m}$. Working backward in time, light traveling from $c$ to $d$ takes time $c\left(t_{m}-t_{4}\right)$. Time $t_{4}$ therefore must satisfy the equation

$$
c\left(t_{m}-t_{4}\right)=\left(x_{R}\left(t_{4}\right)-x_{L}\left(t_{m}\right)\right)
$$

This can be written as an equation in $t_{4}$ using equations (8) and (10),

$$
c\left(t_{m}-t_{4}\right)=\frac{c^{2}}{g}\left[\sqrt{\left(1+\frac{g L}{c^{2}}\right)^{2}+\left(\frac{g t_{4}}{c}\right)^{2}}-\sqrt{1+\left(\frac{g t_{m}}{c}\right)^{2}}\right] .
$$

We again transform this to a dimensionless equation,

$$
T_{m}-T_{4}=\sqrt{(1+\alpha)^{2}+T_{4}^{2}}-\sqrt{1+T_{m}^{2}}
$$


We define the dimensionless parameter

$$
X_{4}=(1+\alpha)^{2}+T_{4}^{2},
$$

which simplifies the equation even further,

$$
\left(T_{m}-T_{4}\right)=X_{4}^{1 / 2}-X_{m}^{1 / 2} .
$$

By squaring, rearranging, and squaring again, we get a polynomial equation in $T_{4}$ of degree 4 ,

$$
\left[X_{4}+X_{m}-\left(T_{m}-T_{4}\right)^{2}\right]^{2}-4 X_{4} X_{m}=0 .
$$

The exact solution of equation (33) is,

$$
T_{4}=T_{m}-\left(\sqrt{1+T_{m}^{2}}+T_{m}\right)\left(\alpha+\frac{1}{2} \alpha^{2}\right) .
$$

Restoring dimensioned quantities we get

$$
t_{4}=t_{m}-\gamma \frac{L}{c}\left(1+\frac{1}{2} \alpha\right)+\beta \gamma \frac{L}{c}\left(1+\frac{1}{2} \alpha\right) .
$$

Light traveling from point $a$ to point $c$ takes time $c\left(t_{4}-t_{2}\right)$. Time $t_{2}$ therefore must satisfy the equation

$$
c\left(t_{4}-t_{2}\right)=x_{R}\left(t_{4}\right)-x_{L}\left(t_{2}\right)
$$

This can be written as an equation in $t_{2}$ using equations (8) and (10),

$$
c\left(t_{4}-t_{2}\right)=\frac{c^{2}}{g}\left[\sqrt{\left(1+\frac{g L}{c^{2}}\right)^{2}+\left(\frac{g t_{4}}{c}\right)^{2}}-\sqrt{1+\left(\frac{g t_{2}}{c}\right)^{2}}\right] .
$$

Following a procedure similar to the one above, we convert this to a dimensionless equation,

$$
T_{4}-T_{2}=\sqrt{(1+\alpha)^{2}+T_{4}^{2}}-\sqrt{1+T_{2}^{2}}
$$

or

$$
T_{4}-T_{2}=X_{4}^{1 / 2}-X_{2}^{1 / 2},
$$

square, rearrange, and square again to get a polynomial equation in $T_{2}$ of degree 4 ,

$$
\left[X_{4}+X_{2}-\left(T_{4}-T_{2}\right)^{2}\right]^{2}-4 X_{4} X_{2}=0 .
$$

The exact solution of equation (33) is

$$
T_{2}=T_{4}-\frac{1}{2}\left[\sqrt{1+T_{m}^{2}}+T_{m}\right]\left[1-\frac{1}{(1+\alpha)^{2}}\right] .
$$


which in dimensioned quantities gives

$$
t_{2}=t_{4}-\gamma \frac{L}{c}\left(1+\frac{1}{2} \alpha\right) \frac{1}{(1+\alpha)^{2}}-\beta \gamma \frac{L}{c}\left(1+\frac{1}{2} \alpha\right) \frac{1}{(1+\alpha)^{2}} .
$$

The total light travel time along the parallel arm is $t_{p a r}=t_{m}-t_{2}$ :

$$
t_{\text {par }}=\gamma \frac{L}{c}\left(2+3 \alpha+2 \alpha^{2}+\frac{1}{2} \alpha^{3}\right) \frac{1}{(1+\alpha)^{2}}-\beta \gamma \frac{L}{c}\left(2 \alpha+2 \alpha^{2}+\frac{1}{2} \alpha^{3}\right) \frac{1}{(1+\alpha)^{2}} .
$$

The difference in light travel times between the perpendicular arm and the parallel arm is $\Delta t=t_{\text {perp }}-t_{\text {par }}=t_{2}-t_{1}$ :

$$
\begin{aligned}
\Delta t= & \gamma \frac{L}{c}\left[\left(2+\alpha^{2}\right) \sqrt{1+\left(\frac{\alpha}{2}\right)^{2}}-\left(2+3 \alpha+2 \alpha^{2}+\frac{1}{2} \alpha^{3}\right) \frac{1}{(1+\alpha)^{2}}\right] \\
& -\beta \gamma \frac{L}{c}\left[2 \alpha+\frac{1}{2} \alpha^{3}-\left(2 \alpha+2 \alpha^{2}+\frac{1}{2} \alpha^{3}\right) \frac{1}{(1+\alpha)^{2}}\right] .
\end{aligned}
$$

This is an exact solution, but it obscures the dependence on the acceleration $\alpha$ because the terms that are lowest power in $\alpha$ cancel. To see the dependence of $\Delta t$ on $\alpha$ we expand the square root and inverse square terms in power series and get

$$
\begin{aligned}
\Delta t=\gamma & \frac{L}{c}\left(\alpha-\frac{3}{4} \alpha^{2}+\frac{5}{2} \alpha^{3}-\frac{185}{64} \alpha^{4}+\frac{7}{2} \alpha^{5}-\frac{2051}{512} \alpha^{6}+\ldots\right) \\
& -\beta \gamma \frac{L}{c}\left(2 \alpha^{2}-2 \alpha^{3}+3 \alpha^{4}-\frac{7}{2} \alpha^{5}+4 \alpha^{6}-\ldots\right),
\end{aligned}
$$

or, keeping only the leading terms,

$$
\Delta t=\gamma \frac{L}{c}\left(\alpha-\frac{3}{4} \alpha^{2}-2 \beta \alpha^{2}\right)+\mathcal{O}\left(\alpha^{3}\right) .
$$

The analysis above has been done in the rest frame. To determine the fringe shift observed by the experimenter in the moving reference frame we need to correct for relativistic time dilation, noting that $\Delta t=\gamma \Delta \tau$, where $\Delta \tau$ is the proper time interval as measured in the experimenter's moving reference frame,

$$
\Delta \tau=\frac{L}{c}\left(\alpha-\frac{3}{4} \alpha^{2}-2 \beta \alpha^{2}\right)+\mathcal{O}\left(\alpha^{3}\right) .
$$

Upon rotating the interferometer through ninety degrees (which doubles the total fringe shift) and using light of proper wavelength $\lambda$, this produces a total observed fringe shift of

$$
\Delta \phi=2 \frac{L}{\lambda}\left(\alpha-\frac{3}{4} \alpha^{2}-2 \beta \alpha^{2}\right)+\mathcal{O}\left(\alpha^{3}\right) .
$$

We note two properties of the fringe shift given by equation (48). First, it reduces to the observed null result when the interferometer is moving at constant velocity $(\alpha=0)$. Secondly, and more importantly, the phase shift depends not only on the rate of acceleration but also on the absolute velocity $(\beta=v / c)$ of the interferometer. 


\section{Feasibility of experimental measurement of absolute velocity}

For easily accessible values of $v$ and $g$ the expected fringe shift according to equation (48) is, unfortunately, quite small. For a comfortable acceleration of $10 \mathrm{~m} / \mathrm{s}^{2}$ and an interferometer arm length of $10 \mathrm{~m}$, the dimensionless acceleration parameter $\alpha$ is approximately $10^{-15}$; for a speed of $370 \mathrm{~km} / \mathrm{s}$ (Earth's speed relative to the cosmic microwave background), the dimensionless velocity parameter $\beta$ is approximately $10^{-3}$; and for visible light the the quantity $2 L / \lambda$ is approximately $3 \times 10^{8}$. For these

values, the three terms in equation (48) are of order $10^{-8}, 10^{-23}$, and $10^{-25}$ fringes respectively. This makes experimental determination of absolute velocity especially challenging, to say the least. Further complicating the problem are the mechanical stresses induced by acceleration which will cause a mechanical compression of the interferometer arm, however this can presumably be measured and compensated for. More extreme values of acceleration, speed, and interferometer arm length could be considered, but it is difficult to imagine an experimental situation in which the velocity dependence of the fringe shift would be large enough to be detectable.

\section{Conclusions}

We conclude that it is possible in principle (though exceedingly difficult in practice) for an observer to measure his speed relative to the absolute reference frame by performing the Michelson-Morley experiment in an accelerated reference frame. This is made possible because of the coupling of velocity and acceleration that results from the asymmetry of the effects of acceleration in a moving reference frame. In other words, because there is an upper limit to achievable speeds, namely the speed of light, it is easier to slow down than it is to speed up. This coupling of $v$ and $a$ is apparent in equation (48), which predicts a non-zero velocity-dependent fringe shift in the Michelson-Morley experiment when performed in an accelerated reference frame.

It should be emphasized that the result obtained here is not in conflict with the limited principle of relativity which says that any experiment performed in a reference frame moving with uniform velocity gives the same results as in a reference frame at rest. It does show, however, that this limited principle of relativity does not extend to accelerated reference frames. On the other hand, the results obtained here are in conflict with the broader philosophical principle of relativity which says that all motion is relative and that absolute motion has no physical meaning. It is the possibility of experimentally disproving this broader principle which makes this result so interesting. 
There is, perhaps, a more fundamental lesson to be learned here. We have considered only one type of experiment, the Michelson-Morley experiment, and shown that, while it fails to detect absolute motion when performed in an inertial frame, it demonstrates velocity dependence when performed in an accelerated frame. This suggests that we should go beyond the large class of experiments that have been considered in inertial frames (which all fail to detect absolute motion) and reconsider performing them in accelerated reference frames. If we are clever enough, perhaps we can find one with enough sensitivity to measure our absolute velocity.

\section{Acknowledgements}

I would like to thank Jackson Messner for a series of interesting discussions on this topic. I would also like to thank two anonymous reviewers whose suggestions have led to a significantly improved paper.

\section{References}

[1] Michelson, A. A., Morley, E. W., "On the Relative Motion of the Earth and the Luminiferous Ether," The American Journal of Science, Vol. XXXIV, 333-341 (1887); reprinted in The World of Physics, vol. 2. Simon \& Schuster, New York (1987), pp. 119-130.

[2] Fitzgerald, G. F., "The ether and the earth's atmosphere," Science 13, 390 (1889); the curious history of Fitzgerald's length-contraction proposal is discussed in Brown (op. cit.).

[3] Lorentz, H. A., "Versuch einer Theorie der electrischen und optischen Erscheinungen in bewegten Körpern," Leiden, 1895; an English translation, "Michelson's Interference Experiment," is in Einstein, et. al., The Principle of Relativity, Dover, New York (1952).

[4] Guerra, V., de Abreu, R., "On the Consistency between the Assumption of a Special System of Reference and Special Relativity," Foundations of Physics 36, 1826-1845 (2006).

[5] Desloge, E. A., Philpott, R. J., "Uniformly accelerated reference frames in special relativity," Amer. J. Phys. 55, 252-261 (1987).

[6] Giannoni, C., Grøn, Ø., "Rigidly connected accelerated clocks," Amer. J. Phys. 47, 431-435 (1979). 
[7] Cahill, R. T., Kitto, K., "Michelson-Morley Experiments Revisited and the Cosmic Background Radiation Preferred Frame," Apeiron 10, 104-117 (2003).

[8] Brown, H. R., Physical Relativity: Space-time structure from a dynamical perspective, Oxford University Press, Oxford (2005).

[9] Barceló, C., Jannes, G., "A Real Lorentz-Fitzgerald Contraction," Foundations of Physics 38, 191-199 (2008).

[10] Møller, C., The Theory of Relativity, 2nd edition, Clarendon Press, Oxford (1972).

[11] Misner, C. W., Thorne, K. S., and Wheeler, J. A., Gravitation, W. H. Freeman, San Francisco (1973).

[12] Hamilton, J. D., "The uniformly accelerated reference frame," Amer. J. Phys. 46, 83-89 (1978).

[13] Rindler, W., Introduction to Special Relativity, Clarendon Press, Oxford (1982). 